\title{
System dynamics for social good
}

\author{
Paulo Gonçalves ${ }^{\mathrm{a} *}$ \\ aUniversità della Svizzera Italiana, Institute of Management and Organizations, Lugano, Tl, Switzerland \\ *paulo.goncalves@usi.ch
}

\begin{abstract}
Paper aims: This paper portrays three examples of system dynamics modes applied to social good.

Originality: System dynamics has a strong tradition of applied research addressing complex social issues ranging from world and urban dynamics to the spread of infectious diseases. System dynamics can provide insight and inform long-term policy in complex social systems. This paper describes system dynamics applications in three important social challenges facing humanity.
\end{abstract}

Research method: In this article, we present three applications of system dynamics research addressing important social challenges, such as HIV/AIDS policy, the eradication of infectious diseases, and capacity building at humanitarian organizations.

Main findings: The examples illustrate how system dynamics can effectively capture key elements of complex dynamic systems allowing managers to understand the behavior of such environments over time.

Implications for theory and practice: This paper contributes to Jay Forrester's bold vision of aiming system dynamics to model important social challenges faced by humanity.

\section{Keywords}

System dynamics. Social good. Case studies. Simulation. Mathematical modeling.

How to cite this article: Gonçalves, P. (2019). System dynamics for social good. Production, 29, e20190062. https://doi. org/10.1590/0103-6513.20190062.

Received: June 24, 2019; Accepted: July 1, 2019.

\section{Introduction}

A social good is a "good" that has positive benefits to society. Examples of social goods include clean air, safety, peace, public libraries, accessible education, and universal healthcare, among many others. A social good is also known as "common good", a shared good that is beneficial for society (Lee, 2013). The notion of common good arises in Ancient Greece. Plato, in The Republic, recognizes that to achieve social justice, individuals should be taught to pursue the common good (Plato, 2004). According to Aristotle (1998), the common good involved a benefit to a collection of individuals (e.g., citizens of a nation, or a city-state) and had higher value than an individual good, consisting of human flourishing. The concept of the common good is a consistent theme in political philosophy through the centuries (Lee, 2013).

Since it was founded sixty years ago, system dynamics has featured in multiple articles and books that have embraced problems of a social nature or common good type problems. The founder of system dynamics, Jay Forrester, "[...] dared us to have courage, be bold, and work on the most important challenges facing humanity" (Sterman, 2018, p. 40). In his 1971 "Counterintuitive Behavior of Social Systems" article, Forrester (1971a, p. 109) stated that:

This paper addresses several social concerns: population trends; quality of urban life; policies for urban growth; and the unexpected, ineffective, or detrimental results often generated by government programs. Society becomes frustrated as repeated attacks on deficiencies in social systems lead only to worse symptoms. [...] Because dynamic behavior of social systems is not understood, government programs often cause exactly the reverse of desired results. The field of system dynamics now can explain how such contrary results happen. 
Forrester's (1971a) paper built upon a solid foundation of previous socially minded work, such as Urban Dynamics (Forrester 1969) and World Dynamics (Forrester, 1971b). In Urban Dynamics, Forrester identified the complex structure responsible for urban growth and decay. The book brought forward a very controversial conclusion: that the typical urban improvement policies did not work. Instead, urban improvement programs, such as low-cost housing, job training, and financial aid, were potentially harmful for cities, since they attracted more jobless people, increasing the population and the financial burden on the city, leading to higher taxes on the underemployed. In World Dynamics, Forrester captured with five stocks (i.e., population, industrialization, resources, agriculture and pollution) the complex interactions responsible for global growth and potential collapse. The work identified limits to the continued growth of economies around the world. It highlighted the implausibility of continued growth in a limited planet and kick-started the field of global modeling.

In the remainder of this paper, we illustrate system dynamics as a methodology to model and understand complex social systems. We focus on a few cases addressing problems with a social good component.

\section{Applications of system dynamics for social good}

In this section, we present a few examples of research employing system dynamics as the main methodology to address diverse social challenges. The examples cover HIV/AIDS policy, the eradication of infectious diseases, and capacity building at humanitarian organizations. Each case adheres to a structure with the following components: (i) a dynamic overview, providing a sketch of the problem that includes reference modes and time horizons; (ii) model development and description, capturing critical dynamic elements (e.g., delays, stocks, flows, nonlinearities), and interconnections among elements characterizing the feedback processes and dynamic complexity; (iii) main insights and results; and (iv) recommendations and implications. We start with a look at the application of system dynamics to resource allocation in HIV/AIDS policies.

\subsection{Data driven policy design for HIV/AIDS response programs in the lvory Coast (Gonçalves \& Kamdem, 2016)}

\subsubsection{Dynamic overview}

As of 2013, Sub-Saharan Africa hosted 70\% of the globe's HIV infected population, or almost 25 million people. In West Africa, lvory Coast is the country with the highest HIV prevalence, constituting about 3.7\% of its population infected in 2012 (Figure 1). The high prevalence and incidence rates of HIV/AIDS in lvory Coast poses an immense challenge for the country. At the same time, the accurate monitoring of the evolution of the disease offers lvory Coast an opportunity for effective HIV/AIDS policies.

\section{Estimated people living w/ HIV in Ivory Coast}

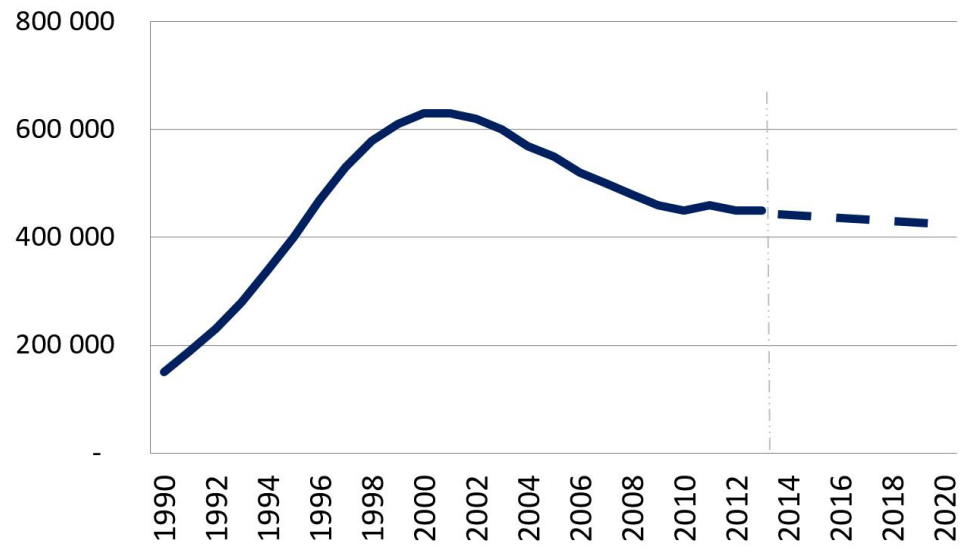

Figure 1. People living with HIV in lvory Coast (Joint United Nations Programme on HIV/AIDS, 2014). 
By tracking the dynamics of the disease with epidemiological models calibrated to country data, the government of lvory Coast and potential donors can better understand the evolution of the disease. By exploring the impact of different health policies in such dynamic models, government officials can make better resource allocation decisions to limit the spread of the disease.

System dynamics offered a way to understand the effectiveness of different policies and the research focused on measuring the impact that different policies could have in curbing the progression of the HIV epidemic in lvory Coast.

\subsubsection{Model development and description}

This research developed the first formal system dynamics model tracking the evolution of HIV/AIDS for lvory Coast. It captured about 25 years of data, calibrating the model to several different variables (starting in 1990) and projecting dynamic behavior through to 2040. In addition to using the model to replicate historical HIV diffusion data, the research informed program design and re-alignment.

There is a significant amount of time series data available on prevalence and incidence rates of HIV/AIDS in lvory Coast for individuals of different age groups. While the data is voluminous, it is fragmented and rarely integrated to support decision making. In addition to time-series data, multiple public reports complement the historical data available. A review of data available in the relevant literature provided another source of data. Finally, we engaged in a number of discussions with decision makers from the lvory Coast Ministry of Health $(\mathrm{MoH})$, the PEPFAR Management Team; one of the authors of the research was an active part of the PEPFAR team. Time series data, public reports and literature data, and insights from decision makers informed our model assumptions, parameters values, and specific model mechanics.

We calibrated the model to individuals of different age groups using historical country data on prevalence and incidence rates for HIV/AIDS from 1990 to 2012. The model, calibrated to 23 years of data for lvory Coast, accurately captured the progression of the HIV/AIDS epidemic until 2012, and then it projected the continued progression 27 years into the future until 2040.

An important aspect of the HIV/AISD model was to properly capture the relevant stocks and flows characterizing the progression of the disease. Because the data included different age groups in the population (e.g., children (0-14 years old); adults (15-49 years old); and elderly (over 50 years old)), it was useful for us to use these different stocks precisely because each group has different characteristics and can be subject to different policies. Because the model captures the dynamics of the disease over 50 years, it is also critical to track the maturing of individuals from childhood to adulthood. Finally, it is essential to show the diffusion of the disease from healthy individuals getting infected, being HIV positive (but asymptomatic), and eventually developing AIDS.

The stock and flow diagram shown in Figure 2 represents the progression of individuals from susceptible through various stages of HIV infection and AIDS, as well as the maturing of individuals from young to adult and elderly. The population susceptible to HIV/AIDS in the model is the adult (mature) population; the majority of HIV infections take place through heterosexual contacts among mature individuals, other HIV infections are due to mother-to-child transmission.

As young healthy individuals become sexually active they mature, entering the group of healthy adults that are susceptible. These individuals leave the adult healthy stock either getting infected, dying, or aging. If infected, they may exit those other stocks due to natural or HIV-related mortality. Figure 2 presents 10 stocks capturing different individuals and stages of the disease. For policy purposes the main stocks include: HIV positive populations, AIDS populations, and populations under antiretroviral therapy (ART), disaggregated by cohorts of children and adults. It also displays the key contagion feedback process driving the diffusion of the HIV/AIDS epidemic.

\subsubsection{Main results and insights}

The model accurately replicates the dynamics of the HIV/AIDS epidemics in lvory Coast. An increase in HIV infection rates in the 90s leads to a rise in HIV positive population. As the population infected with HIV increases, so did HIV contacts, fueling the contagion that spread the disease. With the start of vigorous HIV intervention programs around 2000, lvory Coast experiences a decline in infection rates (Figure 3). From this base line model of the progression of HIVAIDS in the lvory Coast, simulations were run to test the efficacy of various policies to control the disease, individually and in combination. 


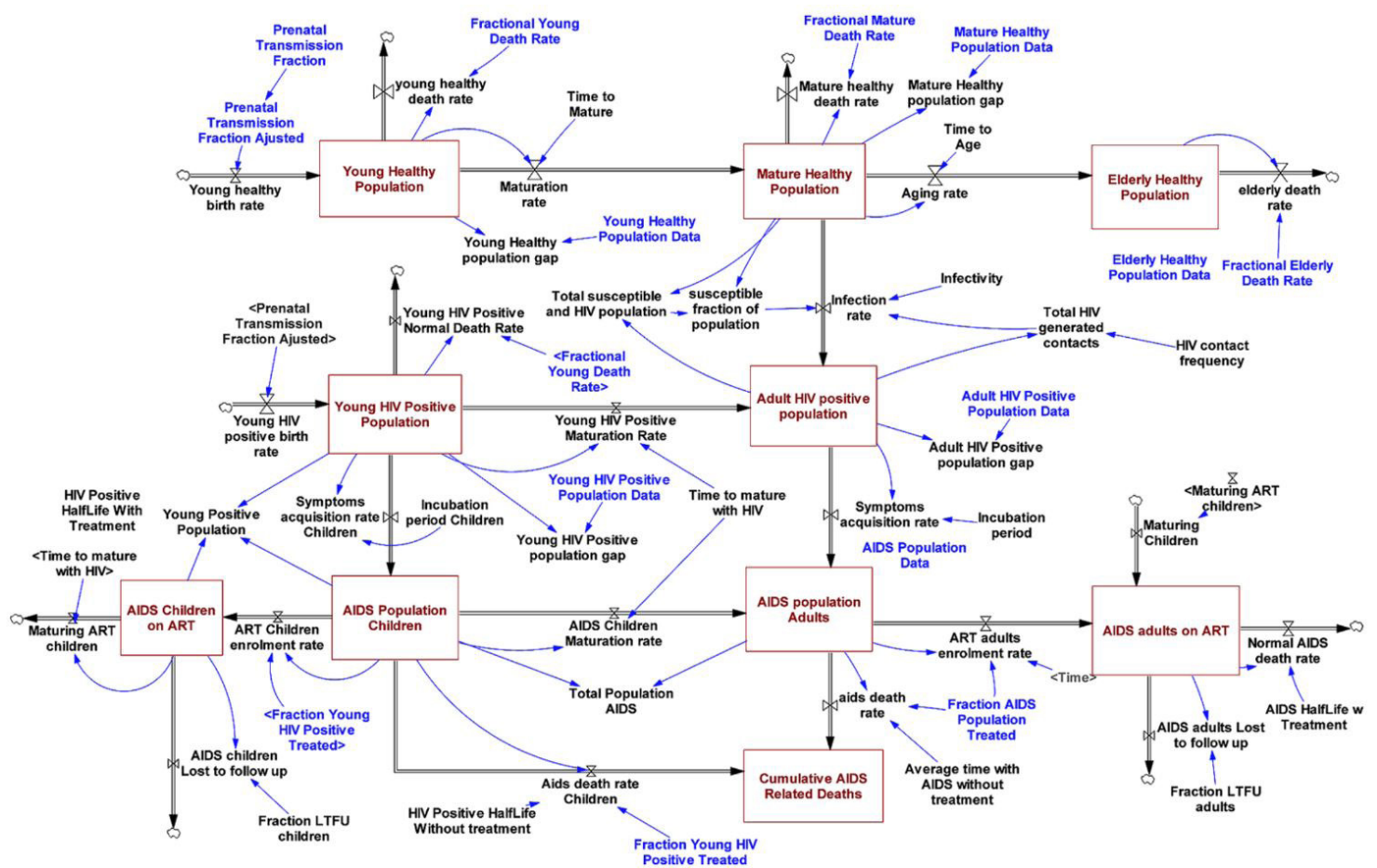

Figure 2. Main stocks and flows capturing the model structure of the progression of HIV/AIDS among the population. Adapted from Gonçalves \& Kamdem (2016).
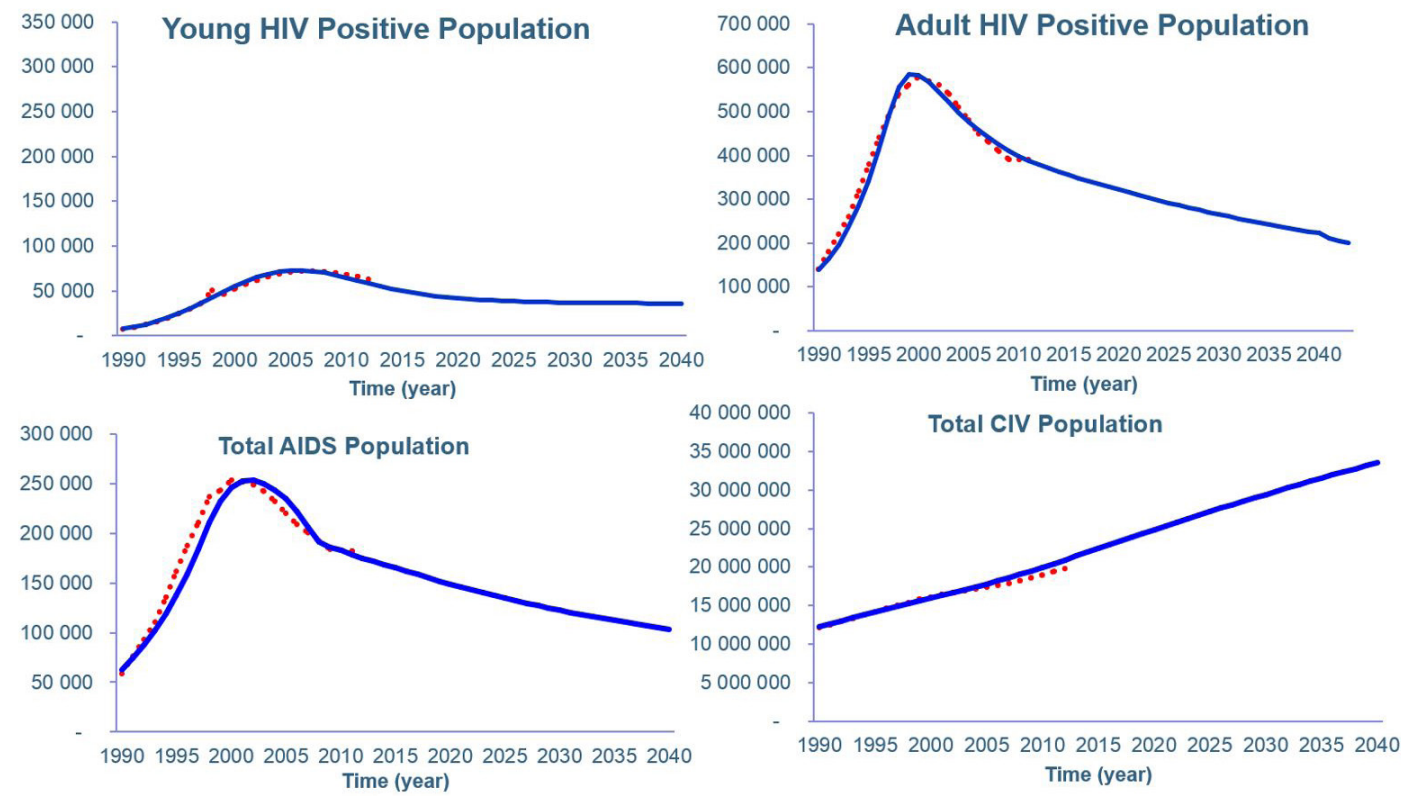

Figure 3. Data and simulations for Young and Adult HIV Positive, AIDS, and Total Population. Adapted from Gonçalves \& Kamdem (2016).

\subsubsection{Recommendations and implications}

Our analyses show that silo policies, addressing single areas (e.g., controlling adult infection rate, HIV prenatal transmission) cannot significantly reduce the overall number of HIV/AIDs infected individuals in the long run. Policy combination are much more effective in reducing HIV/AIDS. The greatest reduction in HIV/AIDS was achieved with the combination of three policies: 
1. Focusing on HIV- Prenatal Transmission;

2. Reducing Infectivity among Adults;

3. Increase in Enrolment Rate in ART.

A policy that combines all three types of interventions at maximum rates would result in the greatest reduction in prevalence. However, such a policy would impose prohibitive costs. Instead, an effective policy would combine fractions of the proposed policies, to capture particular strengths, while mitigating possible weaknesses. An example of such a policy breakdown would be (a) reducing HIV prenatal transmission by $50 \%$; (b) decreasing infectivity among mature healthy by $20 \%$, and (c) increasing adult enrollment rate on ART by $10 \%$.

\subsection{Strategic pathways to eradicate two infectious diseases (Poletaeva, 2018)}

\subsubsection{Dynamic overview}

Allocating resources can be a demanding task, especially when resources are constrained, and insufficient to meet the desired goals. In dynamic settings, when the environment is constantly changing, resource allocation decisions are remarkably difficult. In complex settings, where the interconnection among variables is not clear, resource allocation decisions can have unexpected and unpredictable consequences.

In dynamic and complex settings, decision makers must formulate and implement their new decisions as the environment, and critical information required, is changing. It is common for short-term decisions to generate unpredictable long-term consequences. Managing the spread of infectious diseases represents a class of public health problems where resource allocation decisions are made in a dynamic and complex setting. Decisions by public officials today affect in an unpredictable and unexpected way the future state of the disease. System dynamics can shed light on the long-term consequences of such short-term resource allocation decisions.

Unpredictable disease transmission rates, reporting delays, and delayed response to incidence rates make the management of infectious disease extremely demanding for public officials, most importantly, because each decision can impact the lives of real people. Typically, public officials must allocate scarce resources across multiple infectious diseases threatening the population, considering tradeoffs among them. In the case of a program to eradicate an infectious disease, if resources are withdrawn before the threshold for safe cessation is reached, the disease is likely to reemerge due to the remaining reservoir of infected people. This wavering commitment to an eradication program can lead to a new disease outbreak, reversing the progress achieved by previous efforts.

\subsubsection{Model development and description}

System dynamics based simulators and games have been widely applied to study various aspects of dynamic decision making. Research in medical settings tend to use these simulators as learning tools for educational purposes. However, there is also an opportunity to investigate the dynamic resource allocation decision across multiple infectious diseases. In this research, Poletaeva (2018) creates a learning experiment using Tebbens \& Thompson's (2009) generic model to study how subjects allocate resources across two infectious diseases that compete for limited resources. The experiment captures the allocation of limited funds to vaccinate individuals against each disease. Subjects play the role of public health officials trying to manage the spread of two infectious diseases afflicting the population, with access to limited funds to vaccinate them. The simulator helps researchers gather empirical evidence on strategies that decision makers adopt to manage the diseases. The research sheds light on possible information cues that contribute to certain strategic choices.

\subsubsection{Main results and insights}

Using a system dynamics game based on Tebbens \& Thompson (2009), this research creates the Dynamic Infectious Disease Transmission Model, a typical Susceptible-Infected-Removed (SIR) model arrayed for two diseases (Figure 4). Subjects have access to a pool of funds (e.g., budget) to manage the diseases, however, the total budget is insufficient to eradicate both diseases.

Subjects must decide how to allocate their budget across the two diseases. They are responsible for the "vaccination decision rule". As such, subjects adopt general strategies to manage infections, and the prevalence and mortality associated with each of the diseases. Through the experiment, we gather subjects' resource allocation decisions gaining insight on the factors that contribute to their strategies (e.g., shifting allocation between diseases). 


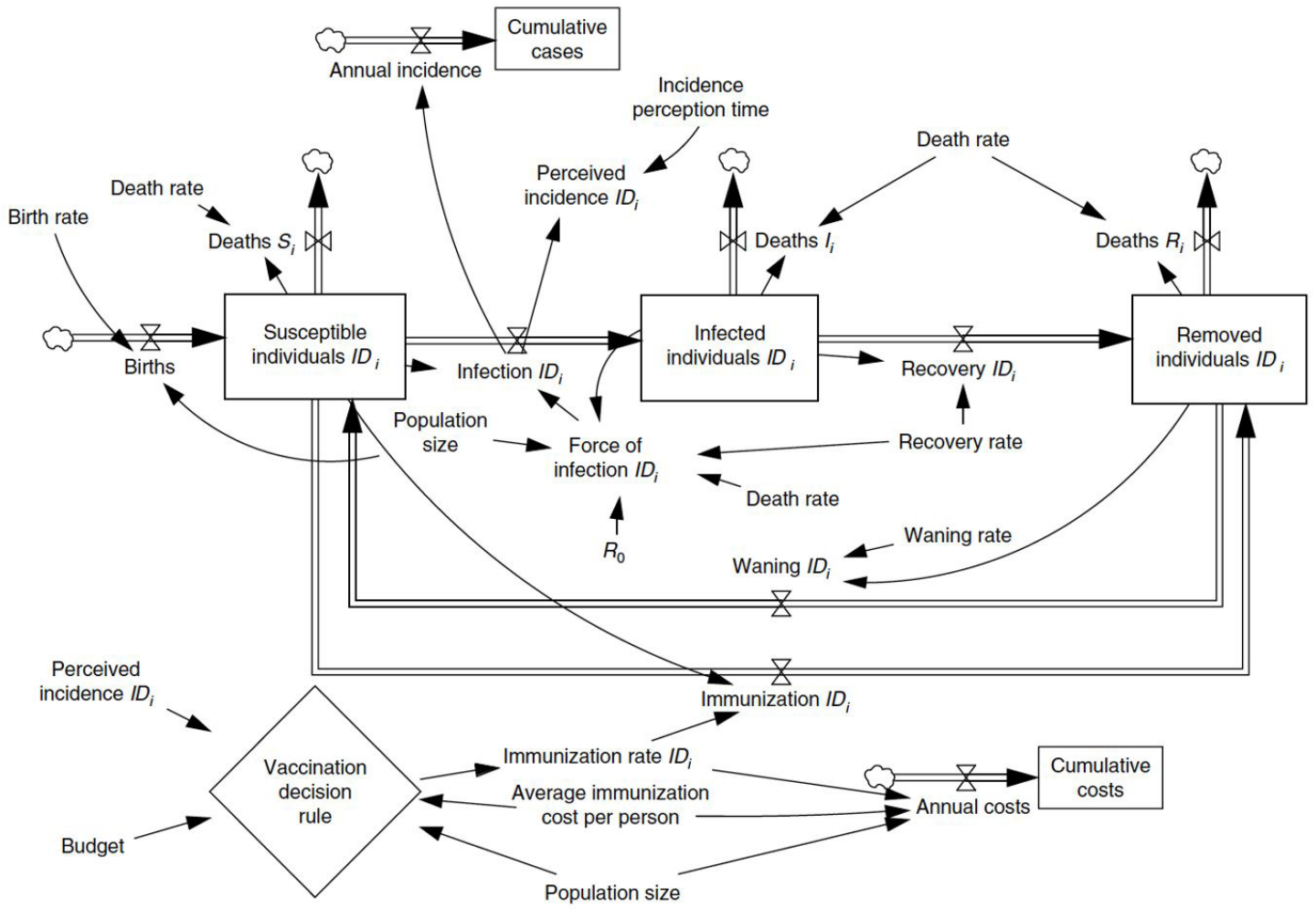

Figure 4. Stock and Flow Diagram for the Dynamic Infectious Disease Transmission Model (Tebbens \& Thompson, 2009).

\subsubsection{Recommendations and implications}

The system dynamics simulator proved to be a fruitful tool for studying behavioral aspects of dynamic decision making. Four general clusters of policies were identified: eradication, wavering, equal allocation, and volatile allocation. The majority of subjects enacted policies in the volatile allocation cluster. That is, subjects did not commit to any definitive strategy; instead, they allocated resources in a volatile and erratic way between the two diseases. Other subjects tried to allocate equal resources to each of the diseases. Some subjects adopted an initial strategy to eradicate one disease by allocating most funds to it; and whatever remained to the "other". This allocation led to decreased incidence rates of the targeted disease; and increased incidence rates to the "other". Over time, when the mortality rates associated with the "other" disease grew high enough, subjects did not manage to maintain their original commitment, wavering the allocation and shifting more funds to the second disease.

Interestingly, in the first round of the game only a few players attempted an eradication strategy, with fewer achieving it. In the second round of the game, however, more players made the strategic choice in favor of eradication. The results suggest that the chance to replay the game facilitated the change in strategy and improvement in performance. However, it is possible that subjects did not develop a deeper understanding of the system and instead simply stumbled into a better policy by chance. Further research can shed light on this open question. Further research can also help understand the issue of commitment towards specific policies.

\subsection{Balancing provision of relief and recovery with capacity building in humanitarian operations (Gonçalves, 2011)}

\subsubsection{Dynamic overview}

Humanitarian organizations (HOs) typically struggle to balance the need to provide relief and the need to build organizational capacity. Every organization must structure its processes, from finance to operations, and from human resources to accounting. HOs must hire and train employees, setup programs, implement projects, 
design information technology systems, structure standard operating procedures, and learn from the field. All these activities require organizational capacity that HOs must build and maintain. At the same time, HOs respond to multiple humanitarian disasters around the world, providing different humanitarian services that range from assessing needs, setting up supply chains, distributing goods, and alleviating suffering. Because providing humanitarian relief represents core activities for humanitarian organizations and requires intense and time sensitive efforts, it tends to receive priority over all other $\mathrm{HO}$ activities.

HOs need to build organizational capacity and, like any other organization, face conflicts with the intensive use of resources to execute their mandates. Providing relief requires HOs to use available resources quickly and effectively, helping them achieve desired results. However, the intensive use of resources also prevents their allocation to activities that build organizational capacity. Clearly, if humanitarian practitioners are providing assistance in the emergency theatre, they cannot attend training programs. If a response requires an operational IT system in the field, the system cannot be undergoing changes and improvements. In summary, intense use of resources to implement and execute day-to-day activities, leaves little opportunity for capacity building efforts. While the tradeoff between exploiting a resource and improving or maintaining it is not new in industry, it seems more challenging when traditional operations involve saving lives and alleviating suffering.

\subsubsection{Model development and description}

This research develops a formal system dynamics model quantifying HOs' tradeoff between capacity building and relief provision Gonçalves (2011). To better understand these tradeoffs, 1 explored the behavior of two extreme strategies: one where HOs use resources to provide relief only and another where HOs only build capacity. The behavior of the two polar extremes shed light on the long-term consequences of such choices. The system dynamics model captures the intended rationality of two main balancing loops (see Figure 5). The Relief/Recovery Focus loop (B1) captures humanitarian managers' short-term approach to allocate more effort to relief to close the gap between people requiring relief and those actually receiving it. The Capacity Focus loop (B2) captures managers' long-term approach to allocate effort to improve organizational capacity to close the same relief gap. Because allocating more effort to relief (e.g., deploying staff to the field) is much faster than building organizational capacity (e.g., training staff), loop B1 yields results faster than loop B2, and typically gets used more often. Furthermore, because resources are limited, more resources allocated to relief result in fewer resources allocated to capacity building, which reinforces the initial strategies selected by managers - captured in the Reinvestment loop (R1).

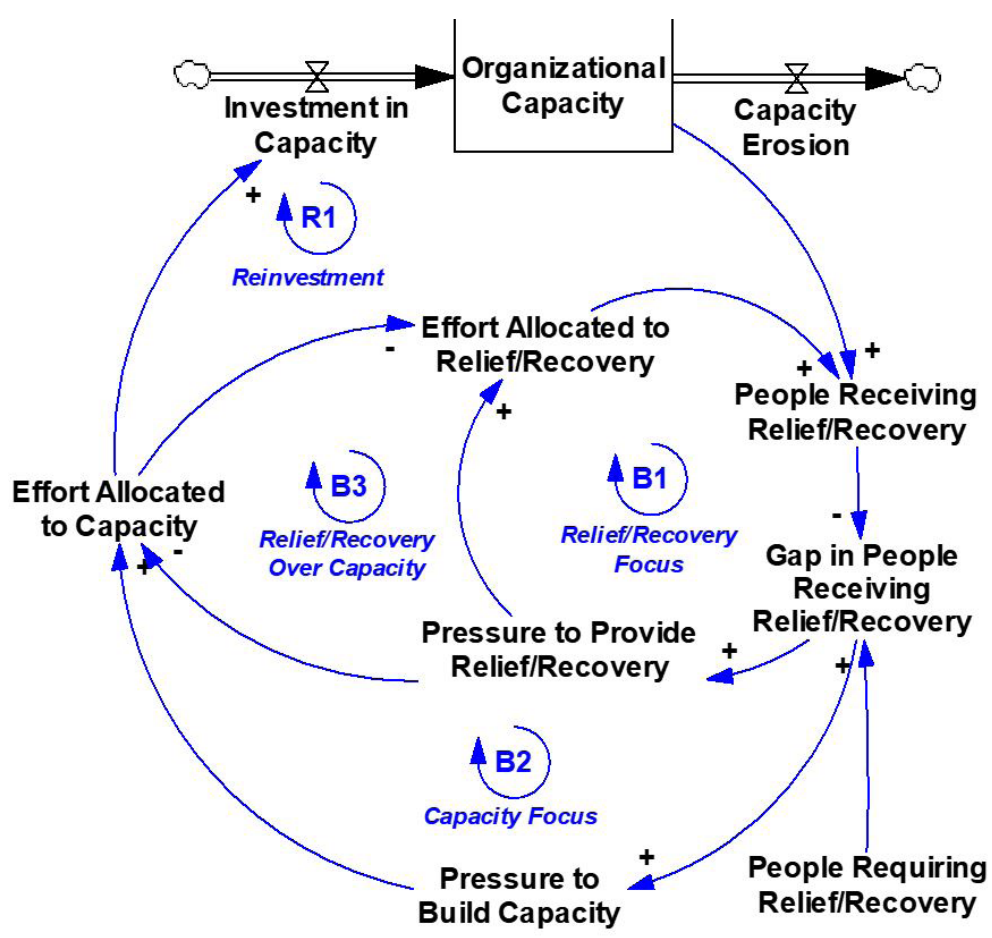

Figure 5. Counterintuitive Behavior from Relief and Capacity Tradeoff (Gonçalves, 2011). 


\subsubsection{Main results and insights}

Figure 6 shows the behavior of key variables including the number of people receiving relief, given by the product of effort allocated to relief/recovery and the productivity of the relief/recovery effort (directly influenced by organizational capacity). Consider the left column of Figure 6, where HOs adopt a relief focused strategy: The HO allocates more effort to relief and due to limited resources, less to capacity building. Relief effort increases quickly, while organizational capacity and productivity of effort decreases slowly. Initially, the number of people receiving relief increases, but over time, with the erosion of organizational capabilities, productivity decreases, and the effectiveness of the operation measured by the number of people receiving relief also decreases. HOs' relief focused strategy shows a better-before-worse behavior. The HO can provide more relief in the short-term, but not in the long-term. In contrast, the right column of Figure 6 captures the performance of HOs adopting an organizational capacity focused strategy. Because the $\mathrm{HO}$ allocates more effort to organizational capacity, productivity of effort increases slowly over time. Due to limited resources, relief effort decreases, leading to a reduction in the short-term relief provided. However, over time, the increase in organizational capability improves productivity, and leads to a more effective operation. HOs' capacity focused strategy shows a worse-before-better behavior. That is, relief provided decreases in the short-term, but increases and thrives in the long-term.

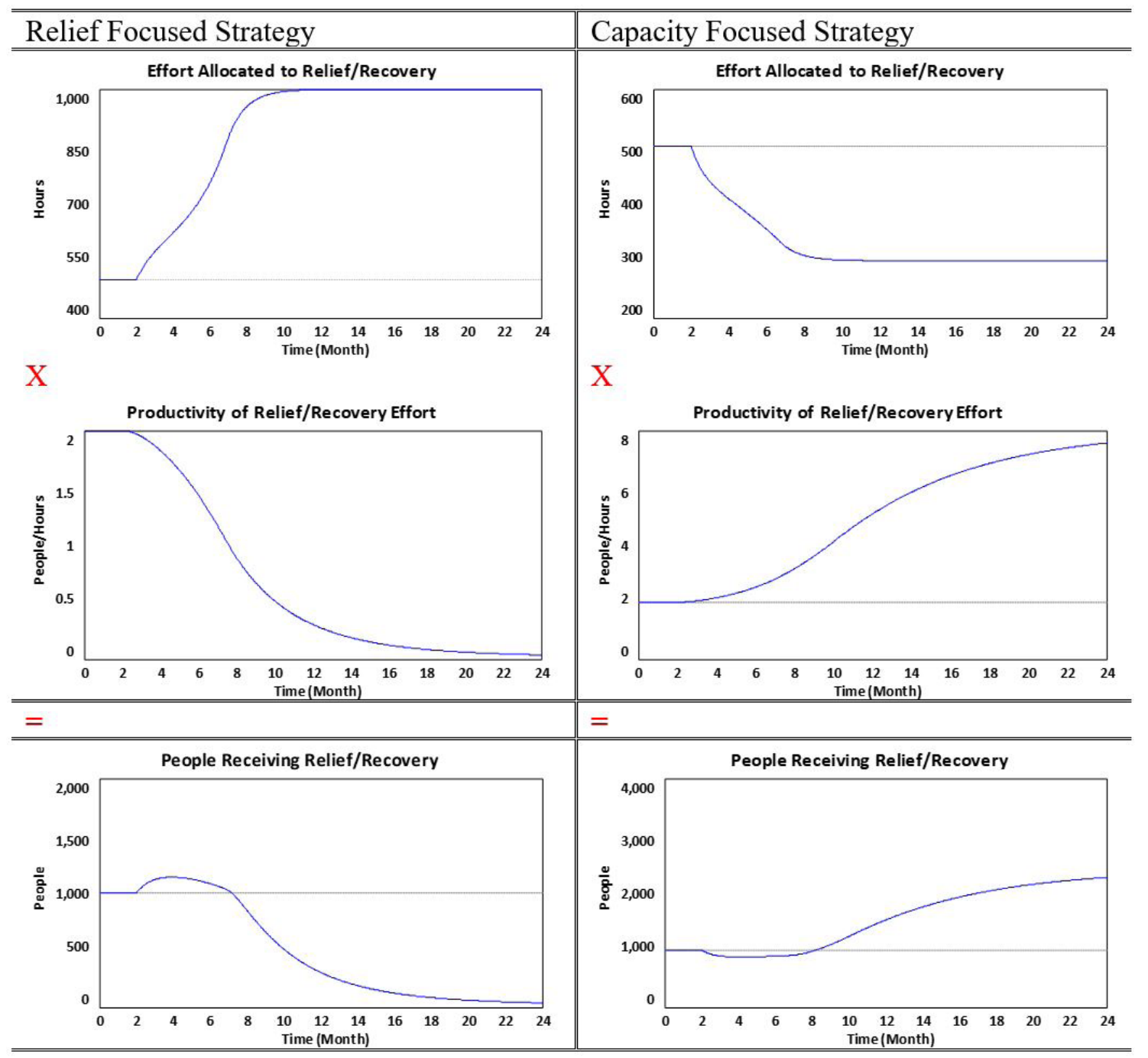

Figure 6. Dynamic Behavior of the Relief and Capacity Focused Strategies (Gonçalves, 2011). 
Because HOs face significant short-term pressure to perform (i.e., provide relief to beneficiaries, alleviate suffering and save lives), it is common to observe HOs adopting a short-term strategy focused on providing relief. However, as relief focused strategies erode organizational capability, it is critical that HOs consider their long-term impact. In contrast, while organizational capacity strategies can improve performance in the long run, they lead to short-term erosion in relief that often prevent HOs from considering them seriously. This is a mistake, and can lead to a capability trap. Before adopting an organizational capability focused strategy, HOs must consider how much and how long they can sustain decreased levels of performance (e.g., number of people receiving relief).

\subsubsection{Recommendations and implications}

The system dynamics approach allows a holistic perspective that includes aspects of HOs strategies combining disaster response in the field and capacity building at the field and headquarters, spanning the short- and long-term impact of resource allocation, and capturing both tangible and abstract variables such as organizational capacity and relief effort. This can enable managers to think more systemically about the theaters where they operate and the dynamic complexity to which they are subjected. Simulation modeling with system dynamics can improve the way managers in humanitarian organizations assess the interactions among variables, experience the long-term side effects of their decisions, and systematically explore new strategies, to develop a more robust understanding of complex systems and more effective strategies.

The research also provides insights into the tradeoff that HOs face between providing relief and building capacity during emergency response.

\section{Conclusion and discussion}

The three examples above show how system dynamics can be used for social good. In particular, they illustrate how system dynamics can effectively capture key elements of complex dynamic systems allowing managers to understand the behavior of such environments and make better decisions over time.

In the case of controlling HIV \AIDS, the model was used to project disease prevalence going 27 years into the future under multiple policy scenarios, allowing managers to make informed decisions about how to allocate their limited budget to get the maximum desired results. Importantly, the model helps prevent unintended consequences by simulating the long term impact of policy decisions on a tested base line that captures the environment and dynamics at play.

In the second example, when faced with the difficult decision of allocating limited funds between two infectious diseases, the model illustrated managerial tendencies towards suboptimal choices. This is not due to managerial incompetence but the difficulty in anticipating outcomes in complex situations. Understanding the interactions of policies over time in a changing evolving setting allows managers to make informed choices about allocating resources to equally important programs.

The third example shows how short-term thinking can ultimately damage an organization's core capacities and provides evidence for how investment in building capacity pays off in the long run. Seeing the effect of decisions in the future via the model, informs managers of potential pitfalls with short-term thinking and the advantages of long-term thinking. In particular, the immediate positive effects of short-term decisions actually chip away at the capacity of the organization to survive.

These and other successful applications of System Dynamics (e.g., Sterman, 2008) share a common thread: they help managers anticipate the long-term effect of their decisions. When using historical data, the modeling effort allows managers to analyze historical information to create a reliable platform upon which projections under various what-if scenarios can be run. When developing behavioral experiments, system dynamics serves as the backbone platform allowing researchers to understand how decision makers think and how dynamic complexity can influence their overall behavior. When dealing with explicit short-term vs. long-term tradeoffs, system dynamics modeling can more explicitly quantify and inform the consequences of current strategies. Altogether, the flexibility and breadth of applications showcase system dynamics as a powerful methodology to address the dynamic complexity of social good environments.

\section{References}

Aristotle. (1998). The politics (C. D. C. Reeve, Trans.). Indianapolis: Hackett Publishing.

Forrester, J. W. (1969). Urban dynamics. Cambridge: MIT Press. 
Forrester, J. W. (1971a). Counterintuitive behavior of social systems. Technology Review, 2(2), 109-140.

Forrester, J. W. (1971b). World dynamics. Cambridge: MIT Press.

Gonçalves, P., \& Kamdem, S. (2016). Reaching an AIDS-free generation in Côte d'lvoire, data driven policy design for HIV/AIDS response programs. International Journal of System Dynamics Applications, 5(1), 43-62. http://dx.doi.org/10.4018/1JSDA.2016010104.

Gonçalves, P. (2011). Balancing provision of relief and recovery with capacity building in humanitarian operations. Operations Management Research, 4(1-2), 39-50. http://dx.doi.org/10.1007/s12063-011-0045-7.

Joint United Nations Programme on HIV/AIDS - UNAIDS. (2014). Narrative report. Geneva: UNAIDS. Retrieved in 2019, June 12, from http://www.unaids.org/en/dataanalysis/knowyourresponse/countryprogressreports/2014countries/CIV_narrative_report_2014.pdf

Lee, S. (2013). Common good. In Britannica Group, Encyclopedia Britannica. Chicago. Retrieved in 2019, June 12, from https://www. britannica.com/topic/common-good

Plato. (2004). Plato's the republic. Indianapolis: Hackett Publishing.

Poletaeva, 0. (2018). Strategic pathways to eradicate two infectious diseases (Master's thesis). European System Dynamics Master Program, Norway.

Sterman, J. D. (2008). Risk communication on climate: mental models and mass balance. Science, 322(5901), 532-533. http://dx.doi. org/10.1126/science.1162574. PMid:18948524.

Sterman, J. D. (2018). System dynamics at sixty: the path forward. System Dynamics Review, 34(1-2), 5-47. http://dx.doi.org/10.1002/ sdr. 1601.

Tebbens, R. J. D., \& Thompson, K. M. (2009). Priority shifting and the dynamics of managing eradicable infectious diseases. Management Science, 55(4), 650-663. http://dx.doi.org/10.1287/mnsc.1080.0965. 